\title{
Microchimerism after heart transplantation: prevalence, predisposing factors, natural history, and prognosis
}

\author{
M.G Crespo-Leiro, M Hermida-Prieto, J.A Rodriguez, J Muñiz, S Barral, M.J Paniagua, \\ L.F Hermida, A Juffé, A Castro-Beiras
}

\begin{abstract}
The success of heart transplantation (HT) is still marred by the need for chronic immunosuppression using agents that display not only limited efficacy and specificity but also considerable toxicity. ${ }^{1}$ Overall 1 -year survival is only $81 \%$, and patient half-life (the time at which the survival rate has dropped to $50 \%$ ) is 9.8 years. $^{2}$ The induction of tolerance to transplanted organs, might make transplantation safer as well as more successful.

Certain studies of recipients of solid organs, including liver, ${ }^{3}$ and ${ }^{4}$ kidney, ${ }^{5}$ lung, ${ }^{6}$ and heart ${ }^{7}$ seem to be associated with apparent induction of tolerance and with the persistence of low levels of donor cells of bone marrow origin (microchimerism). ${ }^{8}$ Other studies, however, have not found an association between microchimerism and tolerance, ${ }^{9}$ and ${ }^{10}$ and there have also been cases of apparently tolerant recipients who do not show detectable microchimerism. ${ }^{11}$ These discrepancies may be explained by differences in the prevalence of microchimerism depending on the organ type and the time after transplantation. ${ }^{12}$ and ${ }^{13}$ Despite these controversies, some workers have attempt to increase the possibility of tolerance by induction enhancing microchimerism via donor bone marrow infusion (DBMI) at the time of transplantation. ${ }^{5}$ and ${ }^{14}$ The work described herein includes studies of the prevalence and natural history of microchimerism after HT, as well as predisposing factors to establish the state, and its relevance to the outcomes of survival, rejection and steroid requirements.
\end{abstract}

\section{Patients and methods}

We determined whether 25 consecutive HT patients exhibited donor microchimerism 3, 6, 12, and 24 months after HT. Immunosuppression was effected with cyclosporin, mycophenolate mofetil, prednisone, and OKT3. The variables examined as possibly predisposing to chimerism were age, sex and weight of donor and recipient, OKT3 dose, and allograft ischemic time. Clinical variables examined as possibly being affected by microchimerism included the number of rejection episodes, total steroid dose, and survival.

Anticoagulated peripheral blood was obtained from donor and recipient before the transplant procedure, and from the recipient 3, 6, 12, and 24 months after HT. Genomic DNA was isolated from leukocytes using a Qiagen kit (Qiagen $\mathrm{GmbH}$, Hilden, Germany) and stored at $-20^{\circ} \mathrm{C}$ until use.

HLA/DRB typing was performed by SSP-PCR (PCR amplification with sequence-specific primers) using 24 primer mixes as described. ${ }^{15}$ and ${ }^{16}$ Before PCR, $5 \mu \mathrm{L}$ aliquots of primer mixes of appropriate concentrations were stored at $-20^{\circ} \mathrm{C}$ in 96 -well reaction plates (Applied Biosystems, Foster City, Calif, USA). At the time of PCR, $5 \mu \mathrm{L}$ of a PCR solution was added to each of the primer mixes, giving final concentrations as follows: $\mathrm{KCl}, 50 \mathrm{mmol} / \mathrm{L} ; \mathrm{MgCl}_{2}, 1.5 \mathrm{mmol} / \mathrm{L}$; Tris-HCI (pH 8.3), $10 \mathrm{mmol} / \mathrm{L}$; glycerol, 5\%; dNTPs, $0.2 \mathrm{mmol} / \mathrm{L}$ each; Cresol Red, $10 \mathrm{mg} / \mathrm{mL}$; DNA, $100 \mathrm{ng}$; Taq polymerase (GibcoLife Technologies, Gaithersburg, Md, USA), 0.4 U. Amplification was performed in a GeneAmp 9700 PCR system thermal cycler (Applied Biosystems) as follows: denaturalization at $94^{\circ} \mathrm{C}$ for 2 minutes, followed by 10 cycles of $94^{\circ} \mathrm{C}$ for 10 seconds and $66^{\circ} \mathrm{C}$ for 1 minute, followed by 24 cycles of $94^{\circ} \mathrm{C}$ for 10 seconds, $61^{\circ} \mathrm{C}$ for 50 seconds, and $72^{\circ} \mathrm{C}$ for 30 seconds. The limit of detection of this technique was between 1 and 10 donor cells per 100,000 recipient cells, as estimated by application to serial dilutions of donor cells within an aliquot of recipient cells bearing a known HLA/DRB haplotype. All PCR products were subjected to electrophoresis for 30 minutes at $250 \mathrm{~V}$ in $1.5 \%$ agarose gels containing ethidium bromide, and were visualized using UV illumination. 


\section{Results}

During the 24 months following HT, microchimerism was at some time detected in 14 of the 25 patients (56\%). The prevalence increased with time after HT, being 8\%, 8\%, 32\% and 40\% at 3, 6, 12, and 24 months, respectively (Table 1). With regard to possible predisposing factors, there was no relationship between microchimerism and recipient or donor sex or age, recipient weight, allograft ischemic time or number of days of OKT3 treatment, but donor weight was on average $11 \mathrm{~kg}$ less among cases with microchimerism than among those without $(P<.01)$; see Table 2$)$. With regard to clinical effects, there was no relationship between microchimerism and number of rejection incidents, total steroid dose, or survival (Table 2). There was one death in the group with chimerism (18 months after HT due to allograft vasculopathy), and one in the group without chimerism (3 months after HT due to lung cancer).

Table 1. Course of Microchimerism Status in the 14 Patients Who Exhibited Microchimerism at Some Time After Heart Transplant

\begin{tabular}{|c|c|c|c|c|}
\hline Patient Number & 3 Months & 6 Months & 12 Months & 24 Months \\
\hline 187 & & & $\mathrm{X}$ & $\mathrm{X}$ \\
\hline 188 & & & $\mathrm{X}$ & $\mathrm{X}$ \\
\hline 190 & & $\mathrm{X}$ & & \\
\hline 199 & & & $X$ & $X$ \\
\hline 200 & & & $X$ & $X$ \\
\hline 205 & $X$ & Exitus & & \\
\hline 206 & & & & $\mathrm{X}$ \\
\hline 210 & & & & $\mathrm{X}$ \\
\hline 213 & & & & $\mathrm{X}$ \\
\hline 214 & & & $\mathrm{X}$ & $\mathrm{X}$ \\
\hline 215 & $\mathrm{X}$ & & $X$ & \\
\hline 218 & & $X$ & & \\
\hline 219 & & & $\mathrm{X}$ & $X$ \\
\hline 222 & & & $X$ & $\mathrm{X}$ \\
\hline
\end{tabular}

Table 2. Factors Investigated as Potentially Affecting or Being Affected by Microchimerism After Heart Transplant: Values in Groups With and Without Detected Microchimerism

\begin{tabular}{lccc}
\hline & Microchimerism $(n=14)$ & No Microchimerism $(n=11)$ & \\
& & $55.8 \pm 8.4$ & .057 \\
Recipient age (y) & $61.9 \pm 4.2$ & $37.6 \pm 8.1$ & .386 \\
Donor age (y) & $33.7 \pm 12.9$ & $74.8 \pm 12.4$ & .833 \\
Recipient weight (kg) & $73.6 \pm 13.7$ & $81.9 \pm 10.1$ & .009 \\
Donor weight (kg) & $70.6 \pm 6.4$ & 90 & .412 \\
Male recipients (\%) & 77 & 80 & .772 \\
Male donors (\%) & 85 & $4.5 \pm 2.3$ & .924 \\
Ischemic time (min) & 150.9 & $1.1 \pm 1.4$ \\
OKT3 (d) & $4.4 \pm 1.8$ & $2300 \pm 1806$ \\
Rejections & $1.5 \pm 1.3$ & $1(9)$ \\
Total steroid dose (mg) & $3404 \pm 2497$ & .894 \\
Deaths (\%) & $1(7.1)$ & .448 \\
\end{tabular}




\section{Discussion}

In animal models of allotransplant ${ }^{17}$ or xenotransplant ${ }^{18}$ the induction of chimerism appears to prolong graft survival. It has been suggested that microchimerism is an important or perhaps essential factor for long-term graft acceptance and tolerance by humans. ${ }^{19}$ As a result, DBMI has been performed at the time of solid organ transplants to augment chimerism with the aim of enhancing graft acceptance and reducing immunosuppression requirement. ${ }^{5}$ The largest DBMI series involving HT patients that we know of was reported by Pham et $\mathrm{al}^{14}$ (when our study was already under way); among 28 patients who received DBMI, the proportion who were free from grade 3A rejection after 6 months was larger than among 24 controls (64\% versus $40 \%$ ), although after 3 years there were no differences between the two groups in either survival or prevalence of coronary artery disease.

It is known that the HT allograft has fewer passenger leukocytes than in the case of liver or lung transplants ${ }^{20}$ which may make it less effective in inducing chimerism. It has also been suggested that treatment with antilymphoid agents might be effective for induction of tolerance. ${ }^{20}$ However, it is not known whether treatment with OKT3 in the immediate post-HT period enhances chimerism. In this study, we found no relationship between the number of days of OKT3 treatment and chimerism.

Series in which microchimerism after solid organ transplant did not appear to benefit allograft tolerance ${ }^{9}$ and ${ }^{10}$ have suggested that in those series in which microchimerism was associated with improved tolerance the association may have been merely circumstantial. ${ }^{21}$ However, the former series9 involved just one determination of microchimerism status per patient; since it has been shown that microchimerism status changes with time, ${ }^{12}$ a single determination is not enough to allow clinical conclusions to be drawn. ${ }^{13}$

In our study, we detected microchimerism in $56 \%$ of our patients at some time during the first 24 months after HT, but prevalence incresased with time from $8 \%$ at 3 months to $40 \%$ at 24 months (Table 1). This increase in prevalence contrasts with the results of Pham et al ${ }^{14}$ who found that the prevalence of chimerism detectable by flow cytometry did not change significantly between 2 and 20 months post-HT in the absence of DBMI ( $40 \%$ and $38 \%$, respectively), and that when DBMI was performed prevalence fell from $85 \%$ at 2 months to $22 \%$ at 20 months.

In this study, most of the variables examined as potentially predisposing factors were not found to be related to microchimerism. The only significant relationship observed was with donor weight, which on average was $11 \mathrm{~kg}$ less among cases with microchimerism than among those without $(P<.01$; see Table 1). Although we have no idea of how donor weight might affect microchimerism, this apparent relationship certainly seems to deserve further examination in studies of larger series of patients.

We also failed to detect any clinical consequences of microchimerism, but in this case the possibility of detection was severely limited by small sample size, since death rates and rejection rates were low in both groups and the variance of total steroid dose was very large (Table 2).

\section{Conclusions}

Microchimerism can be detected in approximately $50 \%$ of HT patients at some time after surgery, the prevalence increasing with time. The clinical relevance of microchimerism is not known with any certainty, nor are the factors that may favour its appearance, although the intriguing significant relationship with donor weight found in this study would seem to deserve further investigation. Until studies with larger numbers of patients throw more light on whether microchimerism does really favor tolerance, treatments aimed at augmenting the former to enhance the latter appear not to be justified.

\section{References}

1. D.M Behrendt, M.E Billingham, M.M Boucek, et al. J Heart Lung Transplant, 10 (1991), p. 841.

2. J Hosenpud, L Bennett, B Keck, et al. J Heart Lung Transplant, 19 (2000), p. 909.

3. T.E Starzl, A.J Demetris, M Trucco, et al. N Engl J Med, 328 (1993), p. 745.

4. T.E Starzl, A.J Demetris, N Murase, et al. Lancet, 339 (1992), p. 1579.

5. T Starzl, A Demetris, M Trucco. Transplantation, 55 (1993), p. 1272.

6. V Kubit, E Sonmez-Alpan, A Zeevi. Human Pathol, 25 (1994), p. 408.

7. Y Masaki, S Hirasawa, G Okuyama. Transplant Proc, 27 (1995), p. 148.

8. D Sachs. Clin Immunol, 95 (2000), p. S63.

9. M Hisanaga, H Schlitt, J Hundrieses, et al. Transplant Proc (1997), p. 1215.

10. K Sivasai, Y Alevy, B Duffy, et al. Transplantation, 64 (1997), p. 427.

11. S Strober, C Benike, S Krishnaswamy, et al. Transplantation, 69 (2000), p. 1549.

12. H.J Schlitt, J Hundrieser, M Hisanaga, et al. Lancet, 343 (1994), p. 1469.

13. E Elwood, C Larse, D Maurer, et al. Lancet, 349 (1997), p. 1358. 
14. S Pham, A Rao, A Zeevi, et al. J Thorac Cardiovasc Surg, 119 (2000), p. 673.

15. A Carter, L Creundolo, M Bunce, et al. Blood, 94 (1999), p. 1471.

16. M Bunce, M Barnardo, et al. Tissue Antigens, 46 (1995), p. 355.

17. T Kawai, B Cosimi, R Colvin, et al. Transplantation, 59 (1995), p. 256.

18. M Tanaka, D Latinne, P Gianello, et al. Transplant Proc, 26 (1994), p. 1326.

19. R Jindal, A Sahota. Postgrad Med J, 73 (1997), p. 146

20. T Starzl, A Demetris, M Trucco, et al. Hepatology, 17 (1993), p. 1127.

21. D Adams, I Hutchinson. Lancet, 349 (1997), p. 1336. 